\title{
Diagnosis and stage of Graves' ophthalmopathy: Efficacy of quantitative measurements of the lacrimal gland based on 3-T magnetic resonance imaging
}

\author{
HAO HU ${ }^{1 *}$, XIAO-QUAN XU ${ }^{1 *}$, FEI-YUN WU ${ }^{1}$, HUAN-HUAN CHEN ${ }^{2}$, GUO-YI SU ${ }^{1}$, \\ JIE SHEN $^{1}$, XUN-NING HONG $^{1}$ and HAI-BIN SHI ${ }^{1}$ \\ Departments of ${ }^{1}$ Radiology and ${ }^{2}$ Endocrinology, The First Affiliated Hospital of Nanjing Medical University, \\ Nanjing, Jiangsu 210029, P.R. China
}

Received April 2, 2015; Accepted April 29, 2016

DOI: 10.3892/etm.2016.3389

\begin{abstract}
The aim of the present study was to determine the efficacy of quantitative measurements of the lacrimal gland based on 3-T magnetic resonance (MR) imaging in the diagnosis and staging of Graves' ophthalmopathy (GO). The study retrospectively enrolled 33 patients with GO (the GO group) and 24 healthy volunteers [the healthy control (HC) group] with orbit MR imaging performed using a 3-T MR scanner. Quantitative parameters of the lacrimal gland, including axial length, axial width, axial area, coronal length, coronal width, coronal area, volume and signal intensity ratio (SIR) of the lacrimal gland to the ipsilateral temporal muscle were measured. The difference of quantitative parameters between the GO and $\mathrm{HC}$ groups, or between active and inactive GO groups were evaluated using a Student's t-test. Receiver operating characteristic analyses were used to evaluate the diagnostic value of the significant parameters in discriminating patients with GO from healthy controls, or discriminating active from inactive GO. All the quantitative measurements of the GO patients were significantly larger than those of the healthy controls $(\mathrm{P}<0.05)$, with the exception of the coronal length $(\mathrm{P}=0.150)$. Axial and coronal width had the best efficacy in discriminating patients with GO from healthy controls. Only SIR was found to be different between the active and
\end{abstract}

Correspondence to: Professor Fei-Yun Wu, Department of Radiology, The First Affiliated Hospital of Nanjing Medical University, 300 Guangzhou Road, Nanjing, Jiangsu 210029, P.R. China

E-mail: wfydd_njmu@163.com

Professor Huan-Huan Chen, Department of Endocrinology, The First Affiliated Hospital of Nanjing Medical University, 300 Guangzhou Road, Nanjing, Jiangsu 210029, P.R. China

E-mail: huanhuanchen2015@163.com

*Contributed equally

Key words: Graves' ophthalmopathy, lacrimal gland, magnetic resonance imaging, diagnosis, stage inactive GO groups $(\mathrm{P}=0.001)$. Setting a SIR of 2.57 as the threshold value, the optimal efficacy was achieved (area under the curve, 0.711; sensitivity, $57.7 \%$; specificity, $77.5 \%$ ) in discriminating between active and inactive GO. Quantitative measurements of the lacrimal gland based on 3-T MR imaging may assist in the diagnosis and stage of GO.

\section{Introduction}

Graves' ophthalmopathy (GO) is an autoimmune disease that accounts for $15-28 \%$ of unilateral exophthalmos and $80 \%$ of bilateral exophthalmos $(1,2)$. In the early stage, patients with GO commonly complain of photophobia, excess tearing, grittiness and foreign-body sensations. Previous studies have indicated that the occurrence of these symptoms is associated with a widened vertical palpebral fissure and increased degree of exophthalmos $(3,4)$. The changes of these anatomic parameters may accelerate the evaporation of tears, and subsequently lead to ocular surface damage and the occurrence of associated symptoms $(3,4)$. However, decreased secretion of tears due to lacrimal gland involvement in patients with GO has also been viewed as a potential cause of associated symptoms in a number of studies (5-7). Therefore, certain researchers have transferred their focus to the lacrimal gland, in attempts to clarify the morphological or functional changes of the lacrimal gland in patients with GO.

Previously, computed tomography (CT) imaging-based studies conducted by Harris et al (8) and Bingham et al (9) found that the morphological measurements of the lacrimal gland in patients with GO were significantly larger than those of healthy controls, which could assist in the diagnosis of GO. However, due to the lower soft tissue resolution and use of ionizing radiation, $\mathrm{CT}$ has been almost completely replaced by magnetic resonance (MR) imaging in the imaging examination of patients with GO. In addition, besides the diagnosis, the accurate staging of GO patients is also very important, considering that different treatment strategies are required for patients with active and inactive GO (10-12). However, studies focusing on quantitative measurements of the lacrimal gland in the staging of GO patients have been lacking. 
Therefore, the present study aimed to determine the efficacy of quantitative measurements of the lacrimal gland in the diagnosis and staging of patients with GO, on the basis of 3-T MR imaging.

\section{Materials and methods}

Patients. The protocol of this retrospective study was reviewed and approved by the institutional review board of the First Affiliated Hospital of Nanjing Medical University (Nanjing, China). The requirement for written informed consent was waived due to the retrospective nature of the study. A retrospective review of the institution's database identified 38 consecutive patients with GO who had undergone MR imaging examination for pretreatment evaluation from June 2013 to December 2014. Among these patients, 33 patients (mean age, $48.4 \pm 13.9$ years; male/female ratio, 12:21) were finally enrolled in the study based on the following inclusion criteria: i) Fulfillment of the criteria of the European Group on Graves' Orbitopathy (EUGOGO) for the diagnosis of GO (13); ii) no prior history of steroid therapy, radiotherapy or surgical decompression; and iii) adequate image quality for imaging assessment. All $33 \mathrm{GO}$ patients had bilateral orbits involved, with a mean disease duration of 7 months (range, 1-24 months).

The assessment of disease activity was performed according to the modified seven-point formulation of the clinical activity score (CAS) (14), including: i) Spontaneous retrobulbar pain; ii) pain on attempted up or down gaze; iii) redness of the eyelids; iv) redness of the conjunctiva; v) swelling of the eyelids; vi) inflammation of the caruncle and/or plica; and vii) conjunctival edema. The CAS is the sum of all items present, and assessment of disease activity was performed for each eye individually. Following assessment by an experienced endocrinologist, eyes with a CAS of $\geq 3$ were accepted into the active stage group; otherwise, they were accepted into the inactive stage group. Finally, a total of 26 eyes were defined as active with a mean CAS of $4 \pm 1$; the other 40 eyes were defined as inactive with a mean CAS of $1 \pm 1$. In addition, 24 healthy volunteers (mean age, 50.0 \pm 12.9 years; male/female ratio, $8: 16)$ were included in the healthy control (HC) group.

MR examination. MR examinations were performed with a 3-T MR scanner (Verio; Siemens Healthcare GmbH, Erlangen, Germany) with a 12-channel head coil. Imaging protocols comprises axial T1-weighted imaging [repetition time (TR)/echo time (TE), 600/10 msec], axial, coronal and sagittal T2-weighted imaging (TR/TE, 3,500-4,000/79 msec) with fat suppression (FS). The other detailed imaging parameters were as follows: Field of view (FOV), 200 mm; matrix, 384x384; slice thickness, $3 \mathrm{~mm}$; gap, $0 \mathrm{~mm}$.

Imaging processing. Due to the difficulty in distinguishing palpebral from orbital lobes on MR imaging, the lacrimal gland was viewed as a whole entity during the measurement $(15,16)$. Quantitative measurements of the lacrimal gland were performed based on both coronal and axial T2-weighted imaging with FS, and the parameters included: i) Axial and coronal length, width and largest area of the lacrimal gland: according to the methods of Huang et al (16), the axial and coronal image in which the lacrimal gland appeared the

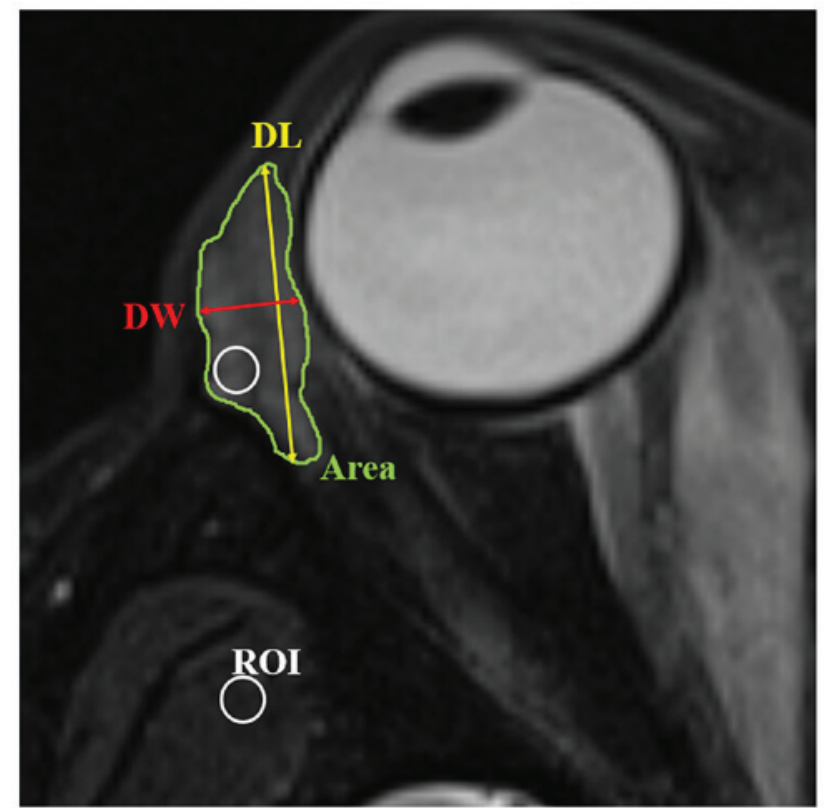

Figure 1. Axial T2-weighted fat suppression imaging showing the methods of measurement of length, width, area, volume and SIR of the lacrimal gland. The axial image in which the lacrimal gland appeared the largest was chosen. Axial area of the lacrimal gland was obtained by manually delineating the gland border (Area). Axial length of the lacrimal gland was defined from the most anterior tip to the most posterior tip (DL). Axial width was measured from the lateral edge to the medial edge at its widest point perpendicular to the length line on the axial images (DW). Volume of lacrimal gland was obtained by a sum-of-area method. The SIR was calculated as the ratio of signal intensity of the lacrimal gland and adjacent temporalis muscle by applying a 'hotspot' ROI (usually $10-15 \%$ of whole cross-sectional area of lacrimal gland). The ROI placed on lacrimal gland demonstrated the portion of relatively higher signal intensity. SIR, signal intensity ratio; ROI, region of interest.

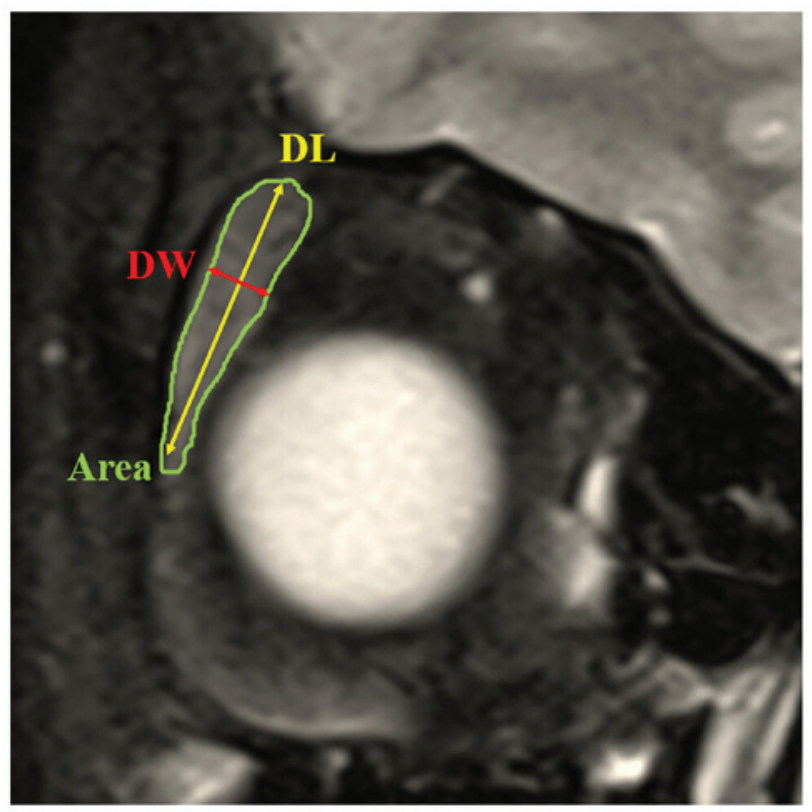

Figure 2. Coronal T2-weighted fat suppression imaging showing the methods of measurement of length, width and area of the lacrimal gland. The coronal image in which the lacrimal gland appeared the largest was chosen. Coronal area of the lacrimal gland was obtained by manually delineating the gland border (Area). Coronal length of the lacrimal gland was defined from the superior tip to the inferior tip (DL). Coronal width of the lacrimal gland was measured from the lateral edge to the medial edge at its widest point perpendicular to the length line on the coronal images (DW). 
Table I. Difference in quantitative measurements of lacrimal gland between the GO and HC groups.

\begin{tabular}{lccr}
\hline Measurement & GO $(66$ eyes $)$ & HC (48 eyes) & P-value \\
\hline Axial width $(\mathrm{mm})$ & $5.7 \pm 3.1$ & $3.8 \pm 0.8$ & $<0.001$ \\
Axial length $(\mathrm{mm})$ & $16.2 \pm 3.5$ & $14.0 \pm 1.9$ & $<0.001$ \\
Axial area $\left(\mathrm{mm}^{2}\right)$ & $68.74 \pm 25.40$ & $43.73 \pm 18.57$ & $<0.001$ \\
Coronal width $(\mathrm{mm})$ & $5.4 \pm 1.0$ & $4.3 \pm 0.7$ & $<0.001$ \\
Coronal length $(\mathrm{mm})$ & $16.2 \pm 3.5$ & $15.3 \pm 2.7$ & 0.150 \\
Coronal area $\left(\mathrm{mm}^{2}\right)$ & $69.18 \pm 22.97$ & $54.96 \pm 19.11$ & 0.001 \\
Volume $\left(\mathrm{cm}^{3}\right)$ & $0.713 \pm 0.300$ & $0.445 \pm 0.194$ & $<0.001$ \\
SIR & $2.49 \pm 0.46$ & $2.17 \pm 0.32$ & $<0.001$
\end{tabular}

SIR indicates the signal intensity ratio of the lacrimal gland to the ipsilateral temporal muscle on fat-suppressed T2-weighted imaging. GO, Graves' ophthalmopathy; HC, healthy control.

largest was chosen. The axial and coronal largest areas of lacrimal gland were obtained using an operator-defined region of interest (ROI) approach (Figs. 1 and 2). The axial length of the lacrimal gland was defined from the most anterior tip to the most posterior tip. The axial width was measured from the lateral edge to the medial edge at its widest point perpendicular to the length line on the axial images (Fig. 1). The coronal length of the lacrimal gland was defined from the superior tip to the inferior tip. The coronal width of the lacrimal gland was measured from the lateral edge to the medial edge at its widest point perpendicular to the length line on the coronal images (Fig. 2). ii) Volume of lacrimal gland: Following the delineation of the border of lacrimal gland in each section on the axial images, the gland volume was obtained from the sum of the areas multiplied by the slice interval (Fig. 1). iii) Signal intensity ratio (SIR) of the lacrimal gland to the ipsilateral temporal muscle: The SIR was calculated based on signal intensity (SI) from fat-suppressed T2-weighted imaging, using the following formula: $\mathrm{SIR}=\mathrm{SI}_{\text {lacrimal gland }} / \mathrm{SI}_{\text {ipsilateral temporal muscle }}$. The SI of the lacrimal gland and the adjacent ipsilateral temporal muscle was measured using a 'hotspot' ROI method (17). The 'hotspot' ROI method meant that the area of the ROI was almost $10-15 \%$ of the whole cross-sectional area of the lacrimal gland (Fig. 1). The ROI placed on the lacrimal gland demonstrated the portion having a relatively higher signal intensity.

A previous study indicated that there is no metrical difference between the left and right lacrimal gland of healthy control subjects (15); therefore, quantitative data of bilateral lacrimal glands in the control group were analyzed together in the present study. The quantitative measurements were performed independently by two neuroradiologists (with 3 and 15 years of experience, respectively), and the mean values of their measurements were used for further statistical analysis.

Statistical analysis. Interobserver agreements between the two radiologists' measurements were assessed using the intraclass correlation coefficient (ICC). Numerical data were averaged and reported as means \pm standard deviation. The Kolmogorov-Smirnov's test was performed to determine whether the quantitative parameters were normally distributed. The Student's t-test and Chi-square test were performed to compare the differences of age and gender between $\mathrm{GO}$ and
HC groups. The Student's t-test was performed to compare all the quantitative measurements of the lacrimal gland between the GO and $\mathrm{HC}$ groups, or between active and inactive GO groups. Receiver operating characteristic (ROC) analyses were further used to evaluate the diagnostic value of the significant parameters in discriminating patients with GO from healthy controls, and discriminating active from inactive GO. $\mathrm{P}<0.05$ was considered to indicate a statistically significant difference. Statistical analysis was performed using SPSS software, version 17.0 (SPSS, Inc., Chicago, IL, USA).

\section{Results}

Comparison of quantitative measurements between the $\mathrm{GO}$ and HC groups. Excellent interobserver agreement was achieved for all quantitative measurements (ICC>0.900). No significant difference was found in either age or gender between the $\mathrm{GO}$ and $\mathrm{HC}$ groups $(\mathrm{P}>0.05)$. All quantitative measurements were significantly larger in the GO group than in the HC group $(\mathrm{P}<0.05)$, with the exception of the coronal length $(\mathrm{P}=0.150)$. Detailed results for the comparisons of all quantitative measurements between the $\mathrm{GO}$ and $\mathrm{HC}$ groups are shown in Table I.

Diagnostic performance of quantitative measurements for discriminating between the GO and HC groups. Axial and coronal width demonstrated the best efficacy in the discrimination of the $\mathrm{GO}$ group from the $\mathrm{HC}$ group (AUC $=0.853$ and 0.810 , respectively). The AUC results that were determined using all the significant quantitative parameters in the discrimination between the GO and HC groups are shown in Table II.

Comparison of quantitative measurements between the active and inactive $G O$ groups. Only SIR demonstrated a significant difference between patients with active and inactive GO $(\mathrm{P}=0.001)$. Detailed comparisons and $\mathrm{P}$-values for the quantitative measurements between patients with active and inactive GO are displayed in Table III. Setting a SIR of 2.57 as the threshold value, the optimal diagnostic value was achieved (AUC, 0.711; sensitivity, 57.7\%; specificity, 77.5\%). The ROC curve using the SIR to discriminate active from inactive GO is shown in Fig. 3. 
Table II. Diagnostic performance of significant quantitative measurements of the lacrimal gland for discriminating between patients with Graves' ophthalmopathy and healthy controls.

\begin{tabular}{lllll}
\hline Measurement & AUC & SE & $95 \%$ CI & P-value \\
\hline Axial width & 0.853 & 0.034 & $0.782-0.920$ & $<0.001$ \\
Coronal width & 0.810 & 0.041 & $0.730-0.890$ & $<0.001$ \\
Axial area & 0.805 & 0.044 & $0.718-0.891$ & $<0.001$ \\
Volume & 0.768 & 0.044 & $0.681-0.854$ & $<0.001$ \\
Axial length & 0.732 & 0.048 & $0.637-0.827$ & $<0.001$ \\
SIR & 0.705 & 0.052 & $0.536-0.740$ & 0.001 \\
Coronal area & 0.681 & 0.051 & $0.581-0.781$ & $<0.001$ \\
\hline
\end{tabular}

AUC indicates area under the curve; SE, standard error; CI, confidence interval; SIR, signal intensity ratio of the lacrimal gland to the ipsilateral temporal muscle on fat-suppressed T2-weighted imaging.

Table III. Difference in quantitative measurements of lacrimal gland between active and inactive Graves' ophthalmopathy.

\begin{tabular}{lccr}
\hline Measurement & Inactive (40 eyes) & Active (26 eyes) & P-value \\
\hline Axial width $(\mathrm{mm})$ & $6.1 \pm 3.8$ & $5.1 \pm 1.1$ & 0.194 \\
Axial length $(\mathrm{mm})$ & $15.9 \pm 3.4$ & $16.7 \pm 3.7$ & 0.363 \\
Axial area $\left(\mathrm{mm}^{2}\right)$ & $68.63 \pm 20.97$ & $68.90 \pm 31.49$ & 0.966 \\
Coronal width $(\mathrm{mm})$ & $5.4 \pm 0.9$ & $5.4 \pm 1.2$ & 0.866 \\
Coronal length $(\mathrm{mm})$ & $16.3 \pm 2.8$ & $16.0 \pm 4.5$ & 0.711 \\
Coronal area $\left(\mathrm{mm}^{2}\right)$ & $68.42 \pm 17.6$ & $70.36 \pm 29.74$ & 0.766 \\
Volume $\left(\mathrm{cm}^{3}\right)$ & $0.737 \pm 0.250$ & $0.676 \pm 0.367$ & 0.425 \\
SIR & $2.34 \pm 0.36$ & $2.72 \pm 0.51$ & 0.001
\end{tabular}

SIR indicates the signal intensity ratio of the lacrimal gland to the ipsilateral temporal muscle on fat-suppressed T2-weighted imaging.

\section{Discussion}

The present study evaluated the efficacy of quantitative measurements of the lacrimal gland, based on 3-T MR imaging, in the diagnosis and staging of GO patients. The results can be summarized as follows. First, the study showed that, with the exception of the coronal length, all other quantitative measurements of the lacrimal gland demonstrated a significant difference between the GO and HC groups. Among these measurements, the axial and coronal width of the lacrimal gland demonstrated the best diagnostic value for patients with GO. Secondly, it was found that the SIR of the lacrimal gland to the ipsilateral temporal muscle based on fat-suppressed T2-weighted imaging might be helpful in staging GO. To the best of our knowledge, the present study was the first to evaluate the efficacy of quantitative measurements of the lacrimal gland, particularly the SIR, in the discrimination between active and inactive GO.

The finding in the present study, that all quantitative measurements of the lacrimal gland in patients with GO, with the exception of coronal length, were significantly larger than those in the HC group, is similar to observations in previous studies $(8,9,16)$. Furthermore, ROC analyses showed that axial and coronal width demonstrated the best diagnostic value for $\mathrm{GO}$ patients. As to the reason, it might be due to the enlargement

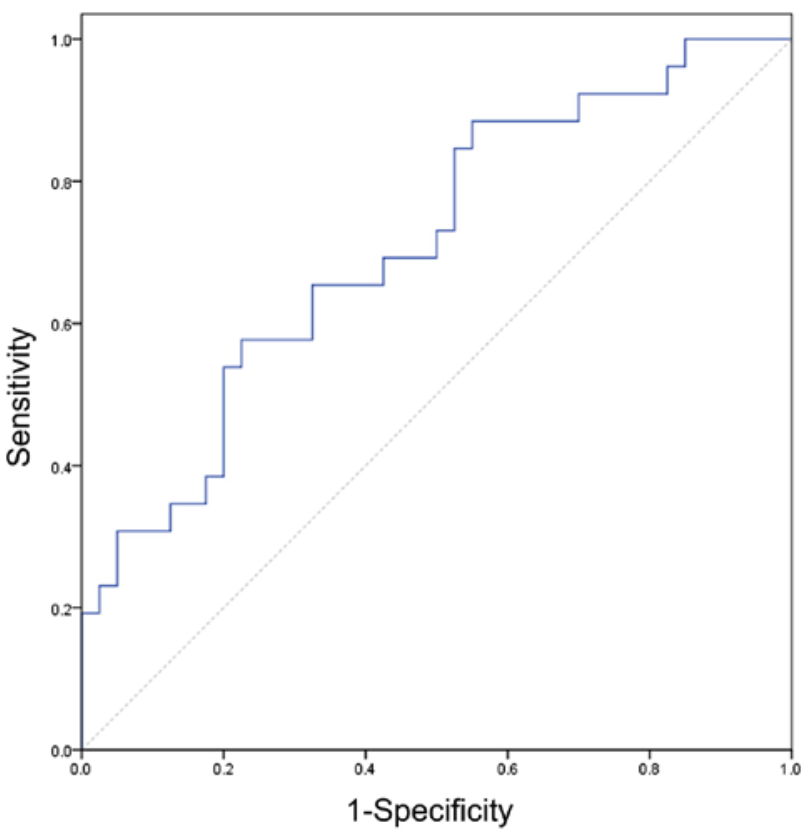

Figure 3. Receiver operating characteristic curve regarding the use of SIR to discriminate active from inactive Graves' ophthalmology. Setting a SIR of 2.57 as the threshold value, the optimal stage value could be achieved (area under the curve, 0.711 ; sensitivity, $57.7 \%$; specificity, $77.5 \%$ ). SIR, signal intensity ratio of the lacrimal gland to the ipsilateral temporal muscle on fat-suppressed T2-weighted imaging. 
of the lacrimal gland in GO patients mainly occurring in the left-to-right direction, but not in the anterior-posterior or up-down direction, as shown by a previous study (16).

Another main finding of the present study was that SIR was the only significant quantitative parameter for distinguishing between active and inactive $\mathrm{GO}$, while the other morphological measurements were not different between the two groups. By contrast, Huang et al reported that two-dimensional measurements of the lacrimal gland differed significantly between active and inactive GO groups (16). However, only 24 GO patients were enrolled in their study, and the relatively small sample size might be responsible for the conflicting results between these two studies. In the present study, SIR was the only significant quantitative parameter for discriminating active from inactive GO. Concerning the possible reason, it is hypothesized that, in the active stage, the main physiopathological changes of the lacrimal gland include inflammatory infiltrations, proliferating fibroblasts and interstitial edema, which would increase the signal intensity of the lacrimal gland on fat-suppressed T2-weighted imaging $(10,12)$. While in the inactive stage, the main physiopathological changes are lacrimal fibrosis, glycosaminoglycan deposition and fatty infiltrations, which would reduce the signal intensity of the lacrimal gland (10-12). Thus, SIR could demonstrate a significant difference between active and inactive GO groups. ROC analysis indicated that an SIR of 2.57 might be the optimal threshold value for discriminating between active and inactive GO. Considering the relatively small sample size, the exact threshold value of SIR requires clarification in a further study with a larger sample size. We consider that our results could be a robust basis for further larger prospective studies.

In the present study, for further usage in a clinical setting, all the MR imaging-based quantitative measurements of the lacrimal gland were performed on the principle of 'simple and convenient', after reviewing several previous studies $(8,9,15,16)$. The image section in which the lacrimal gland appeared the largest was selected for the measurement of gland width, length and largest area, and a sum-of-area method was applied for the measurement of gland volume. All the measurements were easily performed and reproducible. A 'hotspot' ROI approach was used during the calculation of SIR, similar to the method presented by Mayer et al (17). Compared with the whole-area ROI approach, the 'hotspot' method might be less reproducible. However, the 'hotspot' method may better demonstrate focal inflammation, which is crucial in reflecting the local physiopathology of the involved lacrimal gland, particularly considering the heterogeneous changes in the MR imaging of GO patients (10).

The present study has several limitations. First, the sample size was relatively small. However, we consider that the results could be a robust basis for further larger prospective studies. Secondly, the anatomical changes observed by MR imaging were not correlated with functional or pathological changes of the lacrimal gland. Further studies with a larger sample size are needed to associate the anatomical measurements of the lacrimal gland with functional or pathological assessment, so as to clarify the involvement of the lacrimal gland in the pathogenesis of GO from the viewpoint of imaging.

In conclusion, the present study showed that quantitative measurements of the lacrimal gland based on 3-T MR imaging, particularly the axial and coronal width, are able to assist in the diagnosis of GO. Furthermore, the SIR of the lacrimal gland to the ipsilateral temporal muscle based on fat-suppressed T2-weighted imaging might be helpful in staging GO. Therefore, it is suggested that, during the assessment of the MR imaging results of patients with GO, the lacrimal gland should be quantitatively measured, and viewed as a reference standard in the diagnosis and staging of GO.

\section{References}

1. Iyer S and Bahn R: Immunopathogenesis of Graves' ophthalmopathy: The role of the TSH receptor. Best Pract Res Clin Endocrinol Metab 26: 281-289, 2012.

2. Ludgate $\mathbf{M}$ and Baker G: Unlocking the immunological mechanisms of orbital inflammation in thyroid eye disease. Clin Exp Immunol 127: 193-198, 2002.

3. Gilbard JP and Farris RL: Ocular surface drying and tear film osmolarity in thyroid eye disease. Acta Ophthalmol (Copenh) 61: 108-116, 1983.

4. Khurana AK, Sunder S, Ahluwalia BK and Malhotra KC: Tear film profile in Graves' ophthalmopathy. Acta Ophthalmol (Copenh) 70: 346-349, 1992.

5. Chang TC, Huang KM, Chang TJ and Lin SL: Correlation of orbital computed tomography and antibodies in patients with hyperthyroid Graves' disease. Clin Endocrinol (Oxf) 32: 551-558, 1990.

6. Eckstein AK, Finkenrath A, Heiligenhaus A, Renzing-Köhler K, Esser J, Krüger C, Quadbeck B, Steuhl KP and Gieseler RK: Dry eye syndrome in thyroid-associated ophthalmopathy: Lacrimal expression of TSH receptor suggests involvement of TSHR-specific autoantibodies. Acta Ophthalmol Scand 82: 291-297, 2004

7. Jacobson DH and Gorman CA: Endocrine ophthalmopathy: Current ideas concerning etiology, pathogenesis and treatment. Endocr Rev 5: 200-220, 1984.

8. Harris MA, Realini T, Hogg JP and Sivak-Callcott JA: CT dimensions of the lacrimal gland in Graves orbitopathy. Ophthal Plast Reconstr Surg 28: 69-72, 2012.

9. Bingham CM, Harris MA, Realini T, Nguyen J, Hogg JP and Sivak-Callcott JA: Calculated computed tomography volumes of lacrimal glands and comparison to clinical findings in patients with thyroid eye disease. Ophthal Plast Reconstr Surg 30: 116-118, 2014.

10. Bhatti MT and Dutton JJ: Thyroid eye disease: Therapy in the active phase. J Neuroophthalmol 34: 186-197, 2014.

11. Bartalena L, Pinchera A and Marcocci C: Management of Graves' ophthalmopathy: Reality and perspectives. Endocr Rev 21: 168-199, 2000.

12. Tortora F, Prudente M, Cirillo M, Elefante A, Belfiore MP, Romano F, Cappabianca S, Carella C and Cirillo S: Diagnostic accuracy of short-time inversion recovery sequence in Graves' ophthalmopathy before and after prednisone treatment. Neuroradiology 56: 353-361, 2014.

13. European Group on Graves' Orbitopathy (EUGOGO); Wiersinga WM, Perros P, Kahaly GJ, Mourits MP, Baldeschi L, Boboridis K, Boschi A, Dickinson AJ, Kendall-Taylor P Krassas GE et al: Clinical assessment of patients with Graves' orbitopathy: The European Group on Graves' orbitopathy recommendations to generalists, specialists and clinical researchers. Eur J Endocrinol 155: 387-389, 2006.

14. Mourits MP, Prummel MF, Wiersinga WM and Koornneef L: Clinical activity score as a guide in the management of patients with Graves' ophthalmopathy. Clin Endocrinol (Oxf) 47: 9-14, 1997.

15. Tamboli DA, Harris MA, Hogg JP, Realini T and Sivak-Callcott JA: Computed tomography dimensions of the lacrimal gland in normal caucasian orbits. Ophthal Plast Reconstr Surg 27: 453-456, 2011.

16. Huang D, Luo Q, Yang $\mathrm{H}$ and Mao Y: Changes of lacrimal gland and tear inflammatory cytokines in thyroid-associated ophthalmopathy. Invest Ophthalmol Vis Sci 55: 4935-4943, 2014.

17. Mayer EJ, Fox DL, Herdman G, Hsuan J, Kabala J, Goddard P, Potts MJ and Lee RW: Signal intensity, clinical activity and cross-sectional areas on MRI scans in thyroid eye disease. Eur J Radiol 56: 20-24, 2005. 